\title{
Do Clinicians Tell Patients They Have Prehypertension?
}

\author{
Anthony J. Viera, MD, MPH, Fatima Bangura, BS, \\ C. Madeline Mitchell, MURP, Ana Cerna, BS, and Philip Sloane, MD, MPH
}

Background: The clinical utility of the prehypertension label is questionable. We sought to estimate how often patients with prehypertension are being told about it by their primary care clinicians.

Methods: We conducted a cross-sectional study of adult patients visiting practices within the North Carolina Family Medicine Research Network in summer 2008. Non-hypertensive patients were asked whether a doctor or other health care provider had ever told them they had "prehypertension"; a subsample of patients with measured blood pressure (BP) in the prehypertension range was asked the same question.

Results: Of 1008 non-hypertensive patients, $1.9 \%$ indicated being told they had prehypertension. Among a subsample of 102 patients with measured BP in the prehypertension range, $2.0 \%$ indicated being told they had prehypertension.

Conclusion: Few patients who probably have prehypertension are being told about it by clinicians. (J Am Board Fam Med 2011;24:117-118.)

Keywords: Patient-Provider Communication, Prehypertension

Prehypertension is extremely common. In primary care clinics, as many as $40 \%$ of adults who do not have hypertension have prehypertension. ${ }^{1}$ We recently reported that there appeared to be neither harmful nor helpful effects of labeling patients as prehypertensive. ${ }^{2}$ Whether clinicians are actually using the term "prehypertension" with patients has not been specifically examined to our knowledge, although our recent paper suggests that very few patients with prehyperten-

Submitted 9 September 2010; accepted 14 September 2010.

From the Department of Family Medicine (AJV, PS) and the Cecil G. Sheps Center for Health Services Research (CMM, AC, PS), School of Medicine, University of North Carolina at Chapel Hill, Chapel Hill (FB).

Funding: Funding was provided in part by a grant from National Institute of Diabetes and Digestive and Kidney Diseases grant no. T35 DK07386 to the University of North Carolina at Chapel Hill School of Medicine. Data collection and analysis for this project was funded by a National Institutes of Health grant no. K07 AG21587 (PS). Dr. Viera's time was funded by a National Institutes of Health/National Center for Research Resources grant no. 1KL2RR025746-01.

Conflict of interest: none declared.

Corresponding author: Anthony J. Viera, MD, MPH, Department of Family Medicine, University of North Carolina at Chapel Hill, 590 Manning Drive, Chapel Hill, NC 27599-7595 (E-mail: anthony viera@med.unc.edu). sion are told about it. ${ }^{2}$ The purpose of this study was to estimate how often patients with prehypertension are told about it.

\section{Methods}

We conducted a cross-sectional study of patients visiting seven primary care practices within the North Carolina Family Medicine Research Network (NC-FM-RN). ${ }^{3}$ In summer 2008, research assistants offered participation to all adult English and Spanish-speaking patients in waiting rooms of these seven practices across NC during a 3-week period. In addition to demographic and other health questions, participants were asked to indicate whether a doctor or other health care provider had told them they had "high blood pressure or hypertension" (Yes/No), "prehypertension" (Yes/ $\mathrm{No}$ ), or "borderline hypertension" (Yes/No). To estimate the prehypertension "eligible" population, we used an independently validated automatic oscillometric monitor with a cuff that accommodates both standard and large adult arms ${ }^{4}$ to measure blood pressures of a subsample of participants while in the waiting rooms. This study was approved by the Institutional Review Board of the University of North Carolina at Chapel Hill School of Medicine. 
Table 1. Percentage of Nonhypertensive Adult Patients Reporting being Told about Prehypertension or Borderline Hypertension

\begin{tabular}{lcc}
\hline & $\begin{array}{c}\text { All Nonhypertensive Adults in the Sample } \\
(\mathrm{n}=1008)\end{array}$ & $\begin{array}{c}\text { Nonhypertensive Adults with Measured BP in the } \\
\text { Prehypertension Range }(\mathrm{n}=102)\end{array}$ \\
\hline Prehypertension & $1.9(1.0-2.7)$ & $2.0(-0.8-4.7)$ \\
Borderline hypertension & $4.3(3.0-5.5)$ & $3.9(1.0-7.8)$ \\
\hline
\end{tabular}

Values provided as \% $(95 \% \mathrm{CI})$.

$\mathrm{BP}$, blood pressure.

\section{Results}

Of 1754 patients completing the survey (overall response rate $63.9 \%), 42.5 \%$ indicated a history of hypertension. Among those not indicating a history of hypertension ( $\mathrm{n}=1008), 4.3 \%$ indicated being told of borderline hypertension, and $1.9 \%$ indicated being told of prehypertension (Table 1). Less than $1 \%$ indicated both. Of a subsample of 182 patients without known hypertension who had BP measured as part of this study, 102 had BP in the prehypertension range. Among this group, $2.0 \%$ of patients indicated being told they have prehypertension and $3.9 \%$ indicated being told that their BP was borderline (Table 1).

\section{Comments}

Few patients who probably have prehypertension recall being told about it. It is possible that patients were told about prehypertension and just do not recall it, but we think it more likely that clinicians do not use this term with patients. The term "borderline" might be preferred by clinicians, but we found that recollection of being told about borderline hypertension was only slightly more common. One possible explanation for not engaging patients in a discussion about prehypertension is that clinicians do not find it useful in practice. It is possible that clinicians counsel patients about lifestyle modifications that will reduce their chances of developing hypertension without telling them specifically about prehypertension.

The authors thank Katharine Butler, Dax Varkey, Shannon Skinner, and Jessica Taylor, who helped to administer the surveys and collect data.

\section{References}

1. Viera AJ. The new "normal" blood pressure: what are the implications for family medicine? J Am Board Fam Med 2007;20:45-51.

2. Viera AJ, Lingley K, Esserman D. Eeffects of labeling patients as prehypertensive. J Am Bard Fam Med 2010;23:571-83.

3. Sloane PD, Callahan L, Kahwati L, Mitchell CM. Development of a practice-based patient cohort for primary care research. Fam Med 2006;38: $50-7$.

4. Viera AJ, Hinderliter AL. Validation of the HEM780REL with easy wrap cuff for self-measurement of blood pressure according to the European Society of Hypertension International Protocol. Blood Press Monit 2007;12:335-8. 